\section{Internal carotid artery dissection at the supraclinoid portion after severe traumatic head injury in a child}

\author{
JUNYA TSURUKIRI - OSAMU NOMURA • TAIHEI OKITA • \\ SHINJIRO FUKAMI • YUKIO IKEDA
}

JUNYA TSURUKIRI ( $\square)$ OSAMU NOMURA • TAIHEI OKITA Department of Emergency and Critical Care Medicine Tokyo Medical University Hachioji Medical Center 1163 Tatemachi, Hachioji-shi Tokyo 193-0998, Japan Phone: 81-42-665-5611

Fax: 81-3-3342-5687 E-mail: junya99@tokyo-med.ac.jp

SHINJIRO FUKAMI • YUKIO IKEDA

Department of Neurosurgery, Tokyo Medical University Hachioji Medical Center Tokyo, Japan

\begin{abstract}
A 14-year-old boy with severe traumatic brain injury (TBI) and cranial fractures was admitted to our emergency department after a motor vehicle accident. An emergency craniotomy was performed, and traumatic carotid artery (CA) dissection $(t C A D)$ was revealed by cerebral angiography. The patient then underwent close observation in the intensive care unit. Traumatic CADs are difficult to diagnose in the early period after injury, and are associated with serious problems and a high mortality rate. There is still a lack of knowledge surrounding its natural history and treatment options, especially in children. Moreover, it commonly occurs at the cervical internal CA, and ICAD at the supraclinoid portion is quite rare. To the best of our knowledge, this is the first report accompanied by radiological images of the clinical course of $t C A D$ at the supraclinoid portion following severe TBI in a child.
\end{abstract}

Key words: traumatic brain injury, craniotomy, neurosurgery, computed tomography, angiography.

\section{Introduction}

Although cerebral artery dissections are potentially fatal, there is still a lack of knowledge related to their natural history and adequate treatment options. Spontaneous dissections occur most commonly in older populations, but traumatic dissections are more prevalent in younger age groups. (1) Traumatic carotid artery (CA) dissection (tCAD) is a serious problem that results in neurological deficits and a mortality rate of up to $40 \%$. (2) Traumatic CAD at the supraclinoid portion is extremely rare, especially in children. This report details the clinical observation, accompanied by radiological images, of tCAD at the supraclinoid portion after severe traumatic brain injury (TBI) in a child.

\section{Case presentation}

A 14-year-old boy was admitted to our emergency department after a motor vehicle accident (MVA). He had no notable past medical history. On examination, he was restless, had a 7 Glasgow Coma Scale score (E1V2M4), blood pressure of 109/83 mmHg, heart rate of 150 beats/minute, respiratory rate of $24 /$ minutes, and his pupils were $4 \mathrm{~mm}$ in diameter. After receiving initial trauma resuscitation, head computed tomography (CT) showed severe traumatic brain injury (TBI) and cranial fractures (figure 1). Because anisocoria was newly determined at the completion of CT, he was emergently transferred to the operating suite. After craniotomy for a right brain contusion and acute subdural hematoma, decompressive craniotomy was performed and the operation was finished.

After finishing the craniotomy, the patient was immediately transferred to the angiography suite and cerebral angiography was performed in order to evaluate the condition of intracranial internal carotid artery (ICA). Angiography of the right ICA showed the pearl and string sign at the supraclinoid portion of the ICA, and traumatic carotid artery (CA) dissection (tCAD) was suspected. A direct carotid cavernous fistula (CCF) was also observed (figure 2A). The patient was then admitted to the intensive care unit (ICU). Angiography on day 2 of hospitalization showed exacerbation of stenosis at the supraclinoid portion, but this improved to normal after 1 week, and the CCF 
was no longer noted (figures 2B,C). Two weeks after admission, the patient made a good recovery and was discharged from the ICU. 3-D CT angiography (CTA) after 3 weeks revealed no re-stenosis or pseudoaneurysm formation at the supraclinoid portion.

\section{Discussion}

This patient was admitted to the hospital after an MVA, and had a potentially fatal situation due to severe TBI and cranial fractures. The most common mechanism of tCAD in pediatrics is either a direct blow to the neck, or head, or hyperextension, and MVAs are the most common causes of such injury. (1) Traumatic CADs commonly occur at the level of the 1st and 2nd vertebrae, which is the anatomically mobile segment of the ICA. (3) In this case, it was necessary to perform angiography postoperatively in order to evaluate the condition of ICA. This was because the petrosal fracture was transversely fractured, and the fracture line, which penetrated an aperture interna canalis carotici, extended to the base of the skull. Impact to the skull causes an opposing movement of the brain parenchyma, which leads to stretching and shearing effects on the vessels. This can in turn lead to a traumatic endothelial intimal lesion due to a high moment of inertia. CAD, which can lead to thrombosis and occlusion of the anterior, middle cerebral artery or CA, is one of the major causes of ischemic stroke in children. Conversely, causes related to hemorrhagic events are less understood in children.

Angiography is the gold standard for diagnosis, and magnetic resonance angiography (MRA) is also a popular imaging method. CT angiography is increasingly used with less invasive methods. (4) Although the focus should

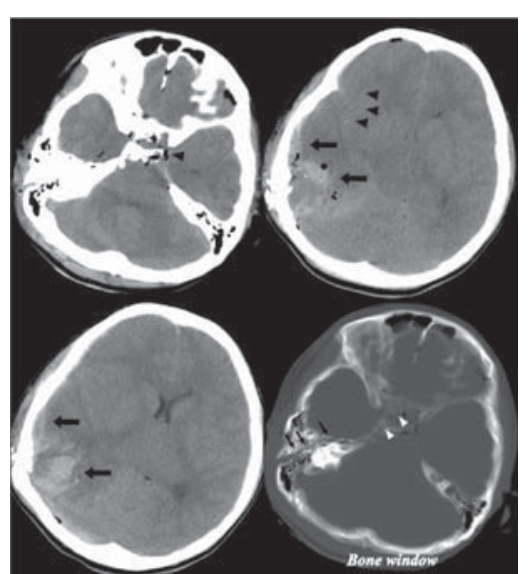

Figure 1. Head computed tomography (CT) shows a right subdural hematoma with a brain contusion at the temporal lobe (large arrow). Pneunoencephalus and subarachnoid hemorrhage are also detected (black arrow head). The bone window in head CT shows a transverse type petrosal fracture (small arrow). The fracture line penetrates an aperture interna canalis carotici and extends to the base of the skull (white arrow head).

be on the natural history of untreated dissections, most reports lack sequential angiographic follow-up, especially in children. The treatment of CAD in relation to the administration of anticoagulation agents, antiplatelet agents and thrombolytics for ischemic symptoms remains controversial due to the presumed increased risk of intracranial hemorrhage.

When fractures involving the base of the

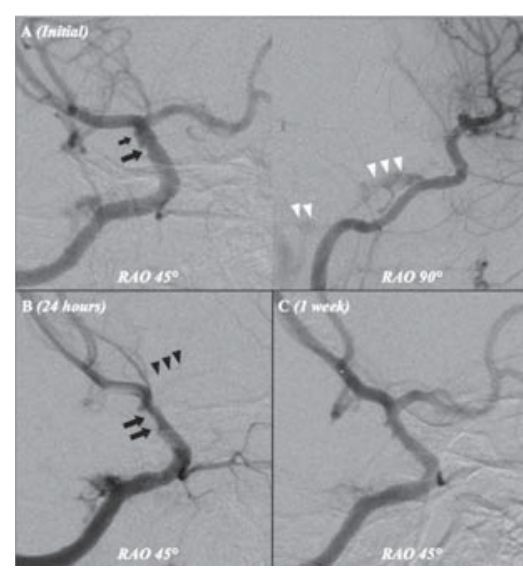

Figure 2. Initial right internal carotid artery (ICA) angiography shows stenosis (large arrow) and dilatation (small arrow) compatible with the žpearl and string sign' at the supraclinoid portion of the ICA. Direct carotid cavernous fistula is also detected (white arrow head). Angiography on day 2 shows stenosis at the supraclinoid portion of the ICA (arrow). Anterior cerebral artery can not be detected (black arrow head). Angiography on day 7 shows complete healing of the dissection without any restenosis of the right ICA. Anterior cerebral artery, anterior choroidal artery and posterior communication artery are detected.

skull are identified in pediatric patients with severe TBI, early evaluation using angiography, MRA, or CTA is essential to confirm details about intracranial ICA injury, especially tCAD, and indicate appropriate treatment, even if no symptoms are present. Observational treatment with intensive care is thus recommended as a conservation option in pediatric patients with severe TBI who undergo tCAD. 


\section{ACKNOWLEDGEMENT}

We sincerely thank Prof. Tamotsu Miki of the Department of Neurosurgery at Tokyo Medical University Ibaraki Medical Center for his assistance.

\section{REFERENCES}

1. Mortazavi MM, Verma K, Tubbs RS, Harrigan M. Pediatric traumatic carotid, vertebral and cerebral artery dissections: a review. Childs Nerv Syst 2011;27:2045-56. doi: 10.1007/s00381-011-1409-x.

2. Larson PS, Reisner A, Morassutti DJ, Abdulhadi B, Harpring JE. Traumatic intracranial aneurysms. Neurosurg Focus 2000;8:e4.

3. Seth R, Obuchowski AM, Zoarski GH. Endovascular repair of traumatic cervical internal carotid artery injuries: a safe and effective treatment option. AJNR Am J Neuroradiol 2012. Epub ahead of print.

4. Lleva P, Ahluwalia BS, Marks S, Sahni R, Tenner M, Risucci DA, et al. Traumatic and spontaneous carotid and vertebral artery dissection in a level 1 trauma center. J Clin Neurosci 2012;19:1112-4. doi: 10.1016/j.jocn.2011.11.018. 